\title{
Kirchhoff based residual moveout migration velocity analysis applied to Marmousi dataset
}

Jadson da S. Lima, (CPGF/UFPA); João Carlos Ribeiro Cruz, (IG/CPGF/UFPA); and Manuel de Jesus da S. Costa, (Campus Abaetetuba/UFPA)

Copyright 2021, SBGf - Sociedade Brasileira de Geofísica

This paper was prepared for presentation during the $17^{\text {th }}$ International Congress of the Brazilian Geophysical Society held in Rio de Janeiro, Brazil, 16-19 August 2021.

Contents of this paper were reviewed by the Technical Committee of the $17^{\text {th }}$ International Congress of the Brazilian Geophysical Society and do not necessarily represent any position of the SBGf, its officers or members. Electronic reproduction or storage of any part of this paper for commercial purposes without the written consent storage of any part of this paper for commercial
of the Brazilian Geophysical Society is prohibited.

\begin{abstract}
One of most important chalenge in seismic exploration methods is to obtain a reliable subsurface earth model of the interested structure in order to find out new oil and gas reservoirs, that in these days are always related to complex geological enviroments. In this work, based on seismic processing free-software, we implement a residual moveout migration velocity analysis algorithm to estimate an accuarate P-wave velocity model useful for efficiently applying in pre-stack depth migration. For each layer is chosen a constant value that represents the initial velocity approximation, and after an established number of iterations, the estimated velocity should be added or subtracted to the previous one until we obtain a value that is sufficiently close to the desired value. In order to verify the reliability of the result, we used as criterium the minimum residual moveout in the common image gathers, which are obtained after migrating the pre-stack seismic data through a Kirchhoff based method. For demostrating the efficiency of the porposed algorithm, we applied it in the Marmousi dataset. By comparing and analyzing the results we achieve a velocity model that provides an accurate seismic image, after application of depth migration method to the pre-stack seismic data with and without random noise. By applying pre-stack depth migration using the estimated model was possible to observe the recovering of the main structures and layers of the well-known Marmousi model. The result of the noisily data tests showed the migration velocity analysis proposed process is very stable.
\end{abstract}

\section{Introduction}

In the seismic imaging we can observe three main problems: low resolution, erroneous shapes and wrong positions of reflectors. With this in mind, migration is considered an essential step in seismic processing, as it corrects certain dips of reflectors to their real positions, thus increasing the spatial resolution of the image. At the end, an image is obtained that is similar to the geological section in depth (Yilmaz, 2001).
In the geophysical literature we find several migration methods, each one presenting its advantages and disadvantages. Among these methods, one of the most traditional and used is the Kirchhoff migration (Schneider, 1978; Bleistein, 1986; Schleicher et al., 1993; Tygel et al., 1996). A determining factor in the success of the migration methods is the correct choice of the velocity field, so-called migration velocity. When it is wrong, the end result will be a distorted image. Conventional methods of updating the migration velocity generally impose one or more of the following limits on the model: homogeneous lateral velocity, small offsets or horizontal reflectors. Such restrictions make it difficult to deal with complex geological environments. In this way, techniques have been developed using the difference of the image depths (residual time) in a set of seismic traces from various offsets or angles that have been adjusted for travel time differences from zero offset (Common Image gathers - CIG) to update migration velocities (Liu and Bleistein, 1995; Liu, 1997; Sarkar and Tsvanki, 2004). Liu and Bleistein (1995), based on the imaging equations derived from Snell's Law, obtained analytical formulas that represent the residual moveout in some cases. Liu (1997) obtained a representation for the derivative of the image depths in relation to the migration velocity. Based on this derived function and the perturbation method, he obtained a formula to update the migration velocity from the residual moveout that is valid for any offset, dip and velocity distribution, which was not achieved in the previous methods. Sakar and Tsvanki (2004) adapted the technique used by Liu (1997) for anisotropic media. Hui (2018) analyzed the factors that affect picks in the residual semblance, such as data quality and geological structure, and proposed solutions and suggestions that reduce or eliminate the impact of these factors at the time of make the pickings.

The main objective of this work is to analyze and compare the results of the migration velocity analysis, based on the study of residual moveout applied to the Marmousi dataset that will be migrated through the conventional Kirchhoff method. The conventional Kirchhoff migration will be based on the work of Schleicher et al. (1993), where it is based on stacking the amplitudes along the diffraction surfaces (or Huygens surfaces). The results of the migrations are organized in Common Image Gathers (CIGs) so that the migration velocity analysis can then be made based on the residual moveout (Liu, 1997), to obtain more accurate velocities and, therefore, images with higher resolution. Tests will also be performed with the addition of noise to the original data to assess the stability of the method. 


\section{Theoretical aspects}

Conventional techniques of the residual curvature analysis method used in the analysis of the migration velocity assume that there is a lateral homogeneity of the velocity and consider that the RMS velocity is equal to the medium velocity (Liu and Bleinstein, 1995), which is not true for layers with lateral velocity anomalies. From the derivative function and the perturbation method, Liu (1997) develops a new formula to update the velocity through the residual moveout, which is valid for any offset, dip and velocity distribution. Thus, the author reviews the residual curvature analysis method that estimates the error in the velocity of the post-migrated data.

First, it is assumed that the surface datum is flat. Then, it is also assumed that the function of the total reflection travel time is known, which, in turn, depends on midpoint and half-offset. For a velocity function $v(x, z)$, therefore, for each half-offset, we have that each point of the reflector in the subsurface is determined by the following equations:

$$
\begin{array}{r}
\tau_{s}\left(x_{s} ; x, z\right)+\tau_{r}\left(x, z ; x_{r}\right)=t(y, h) \\
\frac{\partial \tau_{s}}{\partial y}+\frac{\partial \tau_{r}}{\partial y}=\frac{\partial t}{\partial y}
\end{array}
$$

Where $\tau_{s}$ is the travel time from the source to a point in depth ( $x, z), \tau_{r}$ is the travel time from (x, z) to the receiver and $t(y, h)$ is the total reflection time. If the migration velocity is correct, $\mathrm{z}$ does not depend on h. However, for the wrong choice of migration velocity, there will be dependence of depth $z$ on the half-offset $h$ and, as a result, the image depths in the CIGs will provide information about the desired velocity migration.

It is important to note that Equations (1) and (2) are considered valid even if the migration velocity is wrong and that this system shows a relationship between the imaged depth and the migration velocity. Due to the fact that these equations are not linear, which makes difficult their solutions, Liu (1997) makes use of a mathematical tool called perturbation, which aims to linearize this system of equations through perturbations in the model parameters. For this, it is first necessary to describe the velocity as being characterized by a parameter or a family of parameters:

$$
v=v(x, z ; \lambda)
$$

When $v=v(x, z ; \lambda)=v_{0}+a x+b z, \lambda$ can be $v_{0}, a$ or $b$, Thus, the velocity is determined by estimating parameters instead of velocity itself. We have the following derivative function that relates the imaged depths with the velocity distribution:

$$
g(x, h)=\frac{d z}{d \lambda}
$$

For now the true velocity parameter is $\lambda^{\circ}$, and $Z^{o}$ is the true depth. If there is a small disturbance between the actual parameter and the one used in the migration, this will generate a corresponding disturbance in depth:

$$
\delta z(x, h)=z^{o}-z(x, h) \approx \frac{d z}{d \lambda} \delta \lambda
$$

By using expression (4) we can write the linearized equation,

$$
\delta z=g(x, h) \delta \lambda
$$

In general, we have the true depth $z^{o}$ is unknown. For some authors this problem is solved by using a reference depth concept (Lafond and Levander, 1993), and other authors consider the depth as more one variable to be determined. Liu (1997) developed a new methodology to remove the depth dependence. Since the true depth does not depend on offset, the calculated depths in the CIG for different offsets must be close. This is mathematically express as the minimum variance given by:

$$
\sum_{k=1}^{K} \sum_{j=1}^{m}\left(z_{j}^{(k)}+\delta z_{j}^{(k)}-\overline{\hat{z}^{(k)}+\delta \hat{z}^{(k)}}\right)^{2}=m i n,
$$

where,

$$
\hat{Z}^{(k)}=\left(z_{1}^{(k)}, z_{2}^{(k)}, \ldots, z_{m}^{(k)}\right)^{T}
$$

and,

$$
\delta \hat{z}^{(k)}=\left(\delta z_{1}^{(k)}, \delta z_{2}^{(k)}, \ldots, \delta z_{m}^{(k)}\right)^{T}
$$

In the Equation (7), we have $j$ is the index of offsets within a CIG, and $k$ is the index of the posiction of the CIG on the earth surface. The overline means the mean value of the depths picked in the searched for CIG. And the true depth is approximated by $z^{o} \approx z(x, h)+\frac{d z}{d \lambda} \delta \lambda$.

For the calculation of the relevant derivative function $g(x, h)$, Liu (1997) used the Kirchhoff integral by 
calculating two outputs in the migration, one using the original amplitude and the other using the original amplitude multiplied by the quantity $g(x, h)$ obtained by ray tracing method. Thus, the amplitude ratio of these two outputs will evaluate $g(x, h)$ at the position of the specular source-receiver according to the principle of the stationary phase Bleistein et al. (1987).

If only one parameter is to be determined by the inversion process, the parameter perturbation is given by:

$$
\delta \lambda=\frac{-\sum_{k=1}^{K} \sum_{j=1}^{m}\left(g_{j}^{(k)}-\overline{\hat{g}_{\square}^{(k)}}\right)\left(z_{j}^{(k)}-\overline{\hat{z}^{(k)}}\right)}{\sum_{k=1}^{K} \sum_{j=1}^{m}\left(g_{j}^{(k)}-\overline{\hat{g}_{\square}^{(k)}}\right)^{2}}
$$

If the imaged depths are not close each other, a new itraction is started and stoped when the variance (7) is minimum.

For the implementation of the algorithm, there is a macromodel consisting of several blocks that present different velocities. To determine the velocity in an individual block, a recursive algorithm is used. First, starting from the block closest to the surface, an initial value $\lambda_{i}$ is given, then the migration in depth is performed to obtain the imaged depths and $g_{i}(x, h)$ for a set of CIGs. Then, the perturbation formula is used to update the velocity. With the updated value, a new migration is performed and the the interface is determined. With this interface in hand, the same procedure is repeated for the next block, until all velocities are determined.

\section{Results}

The Marmousi dataset is a very well known 2D complex acoustic data that was based in the Cuanza basin, in Angola used for testing migration algorithms. This model consists of several listric growth fails and innumerable layers that vary in thickness, length and size.

The data consists of 240 common shot gathers with interval between each shot of $25 \mathrm{~m}$. The shots were fired from $3 \mathrm{~km}$ to $9 \mathrm{~km}$. Each shooting family has 96 strokes, with a group interval of $25 \mathrm{~m}$, with the closest distance being $200 \mathrm{~m}$ and the furthest distance being $2750 \mathrm{~m}$. Each trace has 750 time samples, with a $4 \mathrm{~ms}$ sampling interval. The total registration time is $3 \mathrm{~s}$.

The Marmousi velocity model is made up of numerous layers. The minimum velocity is $1500 \mathrm{~m} / \mathrm{s}$ and the maximum is $5500 \mathrm{~m} / \mathrm{s}$. This model has strong lateral and vertical variations. For data processing, a smoothed model called Marmousoft is used, which comes from the original model, and has the important characteristic it has no multiple reflections.

The migration velocity analysis process started from reworking the original velocity model, where the main objective was to join the thinner layers into larger ones, thus making the process more efficient. Initially, about 17 layers were considered, but during the velocity analysis process it was not possible to find satisfactory values for some of them. As a result, the number of layers had to be reduced to 11 (Figure 1) and new tests were carried out. Throughout the procedure, the depths of these layers were not changed and used as a priori information.

With the redesigned model in hand, it was possible to start the velocity analysis process by using the velocity of $1500 \mathrm{~m} / \mathrm{s}$ for all layers. After performing the necessary iterations to estimate the velocity of the first layer, the analysis proceeds to the second layer, and so on. We considered for each individual layer an average of 10 iterations until an appropriate velocity value to be reached and the events in the Common Image Gathers became as flat as possible. It is worth mentioning that the velocity distribution in each layer is a constant function of depth. In Figure 2 we show the final model obtained after the $\mathrm{K}$ MVA method.

We chose two iterations of the velocity analysis for the first layers to show the evolution of flattening the events along the process: The results of the initial iteration and the final one.

In the first iteration, we considered the velocity of $1500 \mathrm{~m}$ for all layers. As expected, the events appear curved upwards, as the velocity used is less than the real one (Figure 3). In the last iteration, we used the velocity 1750 $\mathrm{m} / \mathrm{s}$, and we can note the events are almost completely flats (Figure 4). In Figure 5 we present the $\mathrm{CIGs}$ after application of the estimated end velocity model.

Using the final estimated velocity model, we performed the depth Kirchhoff migration of Marmousi dataset without noise. We noted the result of the velocity analysis provided an efficient recovering of the shallower structures and those that do not present such a complex geology in the initial and final parts of the model. Even in the central part of model with higher heterogeneity, the KMVA method proved to be efficient (Figure 6).

The migration results, after adding noise to the data, using the end estimated velocity model (Figure 7) and the original model (Figure 8) are quite similar. In both cases, it was possible to observe that geological structures are easily identifiable as well as the main layers, not affecting the work of the interpreter.

The tests carried out with the signal-to-noise ratio of 5 showed migration results of highly noisily dataset, affecting in a non-significant way only those less expressive reflectors. The main structures and layers remain easily identifiable. It is concluded, therefore, that the velocity analysis process is stable, robust and that it would be a good option for application in real data.

\section{Conclusions}

By using the K-MVA method presented in this work, we estimate an accurate depth velocity model that after smoothing is applied to depth migration of pre-stack 
seismic data. The K-MVA method starts from an initial appoximation obtained in case of real data from some time to depth conversion strategy, or in the case of synthetic data from a simplified version of the original model. The limits of each layer may be included in the inversion process, that increases a lot the computational efforts. In this paper, we consider as a priori information fixed shapes and limits of layers, and yielded the velocities of each layer to vary only vertically at the specified CMP. By comparing the migrated noisily data using the estimated (Figure 7) and original velocity model (Figure 8), we noted the largest errors in the depth estimate are found in the deeper portions of the central part of model. The distortion in this region probably be due to the complexity of the data and the limitation of the method of solving the small areas of high velocities. Another factor that influences the results is the limitation of the Kirchhof migration algorithm, that is a conventional method and is not robust enough when compared to other more sofisticated migration methods. A final factor to be mentioned is the analysis of the semblance of the residual moveout. The picking of reflectors by semblance measurement becomes much more difficult in complex regions and in the deepest parts.

\section{Acknowledgments}

We would like to thank the support for this research from Petrobras and CPGF of the UFPA.

\section{References}

Bleistein, N., 1986, Two-and-one-half-dimensional inplane wave propagation. attributes. Journal of Seismic Exploration, 10, 19-29.

Bleistein, N., K.J. Cohen, W.J. Stockwell Jr., 1987, Mathematics of multidimensional seismic imaging, migration, and inversion, New York, Spriger-Veerlag. Inc, $510 p$.

Hui Z., 2018, Analysis of Factors Affecting Sobretempo residual Picking and Solutions. International Journal of Oil, Gas and Coal Engineering.,(6), 150-158. doi: 10.11648/j.ogce.20180606.14

Lafond, C.F., and A. R. Levander, 1993, Migration moveout analysis and depth focusing: Geophysics, 58, 91-100.

Liu, Z., and N. Bleistein, 1995, Migration velocity analysis: theory and an iterative algorithm. Geophysics, 60, 142153.

Liu, Z., 1997, An analytical approach to migration velocity analysis. Geophysics, 62, 1238-1249.

Sarkar, D., and I. Tsvankin, 2004, Migration velocity analysis in factorized VTI media. Geophysics, 69, 7087018.
Schleicher, J., M. Tygel, P. Hubral, 1993, 3D true amplitude finite - offset migration, Geophysics, 58 (8), 1112-1126.

Schneider, W.A., 1978, Integral formulation for migration in two and three dimensions. Geophysics, 43, 49-76.

Tygel, M., J. Schleicher, P. Hubral, 1996, An unified approach to 3D seismic reflection imaging - Part II: Theory. Geophysics, 61, 759-775.

Yilmaz, Öz., 2001, Seismic data analysis: processing, inversion, and interpretation of seismic data. Society of Exploration Geophysicists, 2027 p.

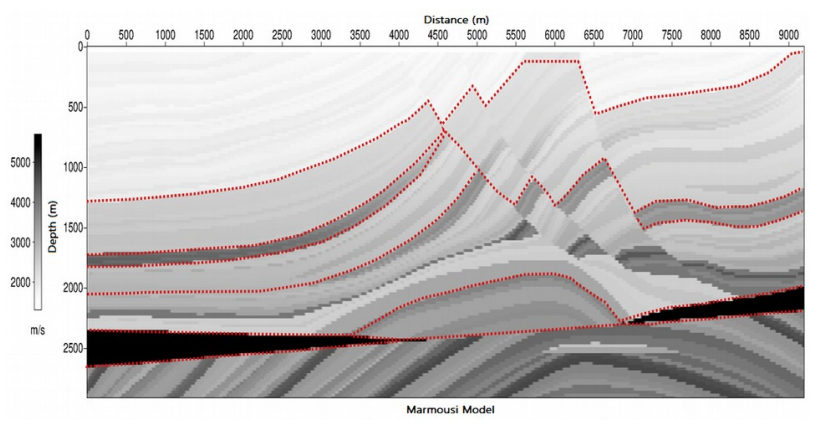

Figure 1: Redesigned structures in red lines of overlaid Marmousi model used as a priori information in the $\mathrm{K}$ MVA method.

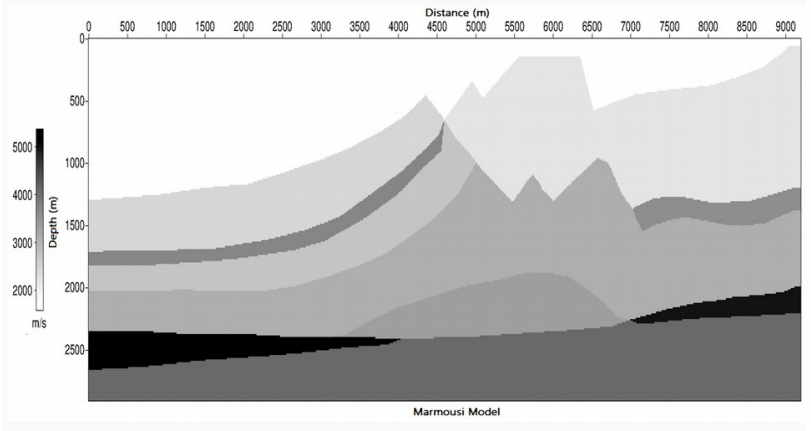

Figure 2: Estimated model after application of the K-MVA algorithm.
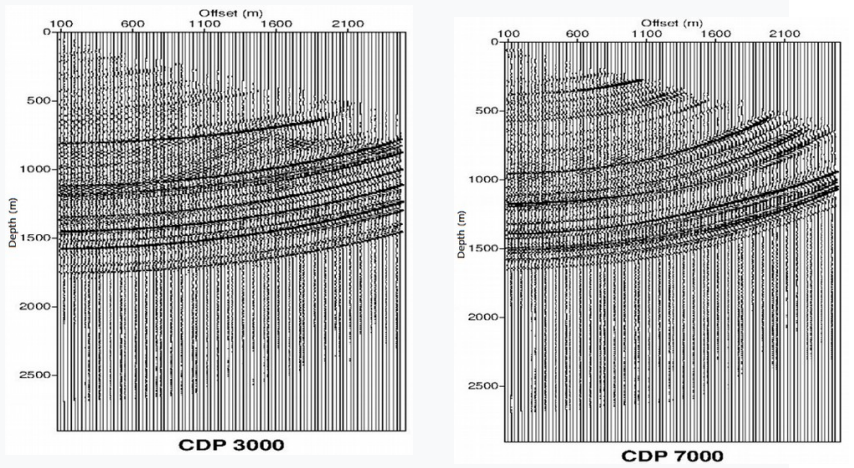

Figure 3: CIGs with velocity $1500 \mathrm{~m} / \mathrm{s}$ for all layers. We can note all reflection horizons are upper ward curved due to the used wrong velocity. 


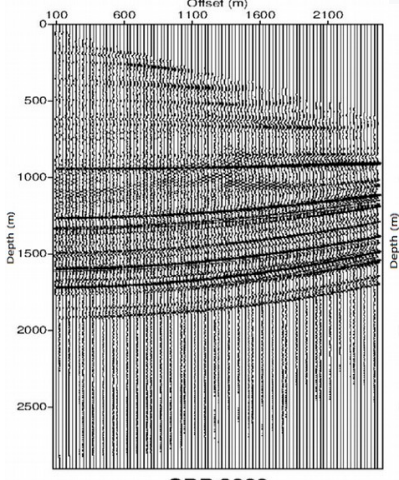

CDP 3000

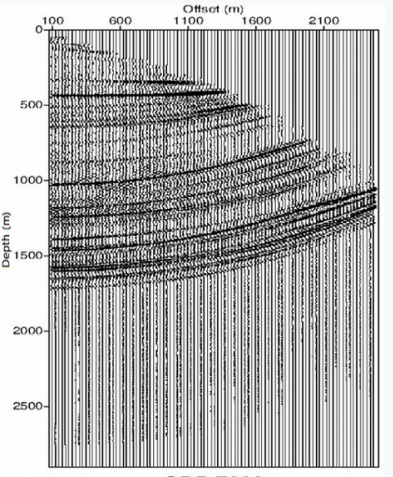

CDP 7000
Figure 4: CIGs with velocity $1750 \mathrm{~m} / \mathrm{s}$ for all layers. We can see in CDP 3000 the reflections above $1000 \mathrm{~m}$ are very well flattened. We can observe the same for reflections above $500 \mathrm{~m}$ in CDP 7000.
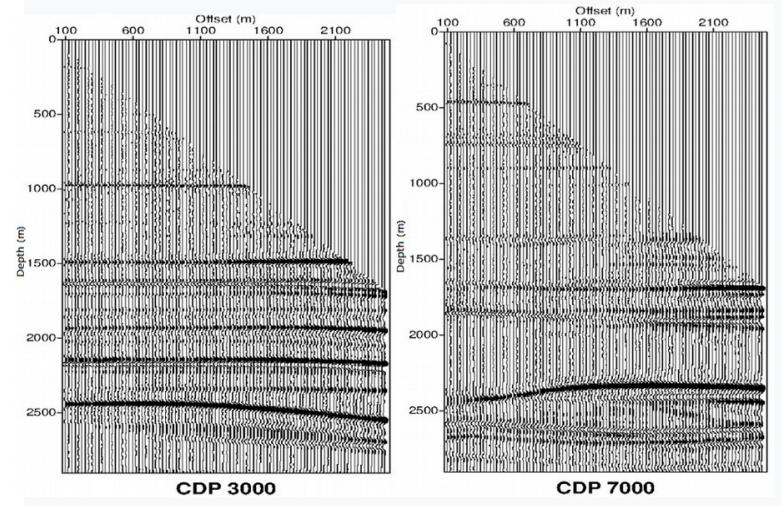

Figure 5: CIGs with estimated end velocity model. We can observe in both CDPs the reflection horizons are well flattened until the depth of $2400 \mathrm{~m}$.

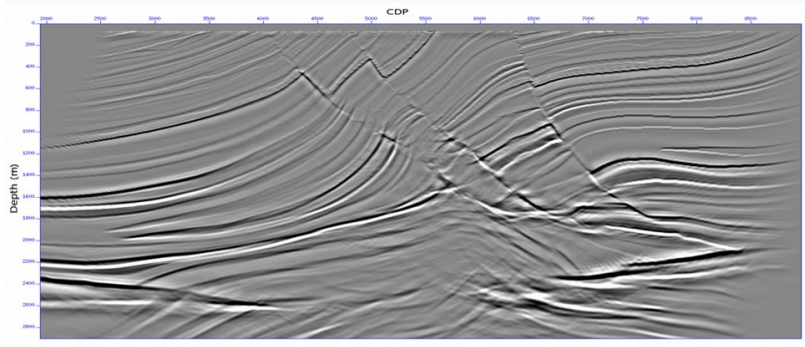

Figure 6: Pre-stack depth migration of the Marmousi dataset without noise using estimated velocity model in Figure 2.

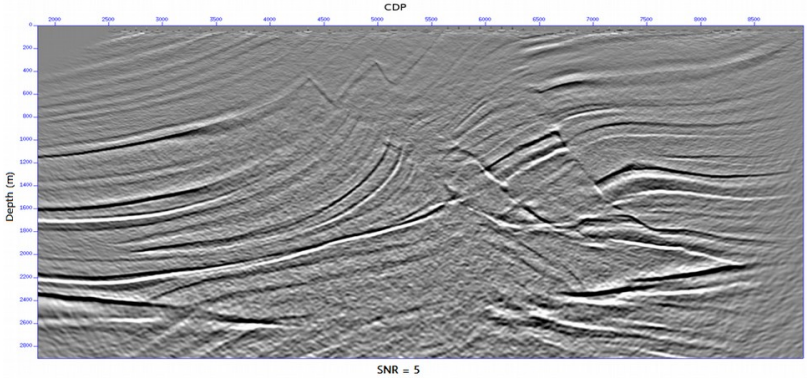

Figure 7: Pre-stack depth Kirchhoff migration of the Marmousi dataset with signal to noise ratio equals 5 using the estimated velocity model in Figure 2.

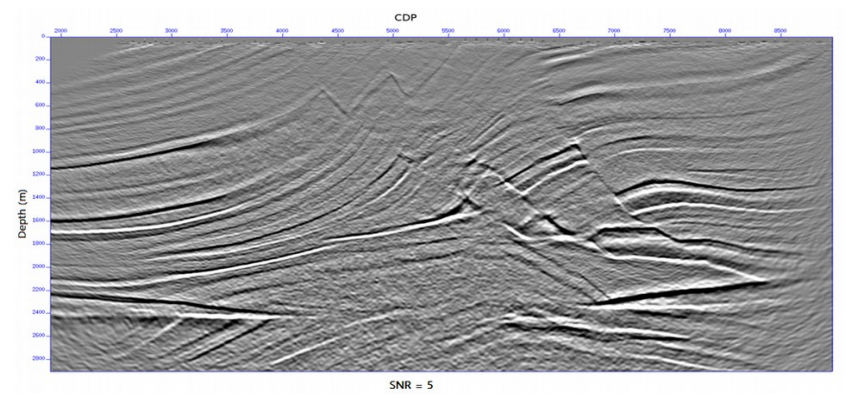

Figure 8: Pre-stack depth Kirchhoff migration of the Marmousi dataset with signal to noise ratio equals 5 using the original velocity model. 